\title{
An Overview of Advanced Chalcogenide Thermo- electric Materials and Their Applications
}

Fiseha Tesfaye $^{1,2}$, Mykola Moroz ${ }^{1}$

1. Ảbo Akademi University, Johan Gadolin Process Chemistry Centre, Piispankatu 8, FI-20500 Turku, Finland

2. Seoul National University, Department of Materials Science and Engineering, Gwanwak-ro 1, 08826, Seoul, Republic of Korea

Abstract: Thermoelectricity is a strong scientific and technological interest due to its wide application ranging from clean energy producing to photon sensing devices. Recent developments in theoretical studies on the thermoelectric (TE) effects as well as the newly discovered thermoelectric materials provide new opportunities for several applications. Though the scale of production is limited, thermoelectric technology provides an alternative to traditional methods of power generation, heating and cooling systems. TE technologies can be used in power generation, heating and cooling applications. They potentially offer significant energy savings through waste heat recovery and augmented cooling. This article critically discusses the current progress in chalcogenide TE materials and the advantages and limitations associated with the TE technologies. The need for new materials discoveries from the point of view of achieving higher figure-of-merit combined with thermal stabilities in intermediate- and hightemperature Peltier and Seebeck effects applications is also emphasized. Besides, this article aims to evaluate the main features of recently characterized multicomponent chalcogenide ionic compounds with high thermal stabilities as potential TE materials to harvest electric power from high-temperature heat flux via thermoelectricity.

Key words: Thermoelectric materials; conductivity; thermal stability; renewable energy

Corresponding author: Fiseha Tesfaye, E-mail:

fiseha.tesfaye@abo.fi

\section{Introduction}

The limited supply of fossil fuels and the growing global population and economic development have caused a steadily increasing price of electricity ${ }^{[1]}$. To address these challenges, renewable energy sources should be developed.

The phenomenon of an electrical current induced by a temperature gradient is termed as "thermoelectricity" [2]. Thermoelectric (TE) effects are based on three transport phenomena: the Seebeck, Peltier and Thomson, which were first observed between 1821 and 1851 , and consequently their applicability to thermometry, power generation and refrigeration was recognized. TE effect can be applied for: (1) TE energy generation when two materials are exposed to different temperatures; (2) TE cooler when the temperature difference is created by transferring heat between two electrical junctions [3]. TE technology has undergone several stages of significant interest as well as research and development, along with periods of inactivity and decline. This is due to the fact that TE has long been too inefficient to be cost-effective in most applications ${ }^{[4]}$. The TE technology developed slowly until the 1930s, thereafter rapid improvements in all areas of TE occurred. In the mid-1960s, practical TE devices emerged for niche applications in aerospace cooling and spacecraft power systems. For example, the radioisotope TE generators were used as power sources in satellites such as Voyager 1 and 2, Pioneer 10 and 11, Apollo 12-17, etc. ${ }^{[5,6]}$. Meanwhile, progress in efficiency improvement was slowed which followed by a steep decline in activity that was to continue for nearly three decades [7]. However, there was renewed interest in TE technology since 1990s due to a combination of factors, notably environmental concerns regarding refrigerant 
fluids, alternative refrigeration and interest in cooling electronic devices ${ }^{[7,8]}$. Contemporary interest in the technology is driven by an increasing awareness of the effect of global warming on the planet earth; a renewed requirement for long-life electrical power sources; as well as the increasing miniaturization of electronic circuits and sensors.

This article presents critical reviews on current progress in chalcogenide TE materials and the advantages and limitations associated with the TE technologies. Furthermore, it highlights the need for new materials discoveries from the point of view of high figure-of-merit combined with thermal stabilities in intermediate- and high-temperature Peltier and Seebeck effects applications. Lastly, it evaluates the main features of new multicomponent chalcogenide ionic compounds with high thermal stabilities as a potential TE material to harvest electric power from high-temperature heat flux via thermoelectricity.

\section{Merits of TE Materials}

TE technology has several advantages, notably it has no moving parts, an ability to function in harsh environments and substantially less maintenance requirements than comparable technologies. When a temperature difference is maintained between two terminals, a thermoelectric module generates clean energy. Given that TE only requires a temperature difference to produce power, these materials can easily generate energy from waste heat streams or other low-grade sources of energy ${ }^{[2]}$. For cooling or refrigeration applications, no chlorofluorocarbons or other materials that require periodic replenishment are necessary, providing a potential environmental benefit with precise temperature control of $\pm 0.1 \mathrm{~K}^{[8,9]}$. The same TE device can be used for heating or cooling and can cool below $273 \mathrm{~K}$. The modules are relatively small in size and weight ${ }^{[10]}$ and can be mounted in any orientation as they are not position dependent ${ }^{[9]}$. With ever increasing power and packaging density of electronic devices, thermal management has become one of the major issues to ensure the performance and reliability of electronic devic$\mathrm{es}^{[11]}$. TE micro-coolers using thin film proce-sses can be an effective solution to thermal manage-ment of electronic devices due to their advantages, such as large cooling power density inversely propor-tional to the thin-film thickness, compatibility with electronic device fabrication and short response time. While various processing techniques can be used for TE thin-film fabrication, electrodeposition is more attractive because it is a rapid and inexpensive me- thod $^{[11]}$. Furthermore, TE devices are quiet in operation and do not release emissions and can be considered an environmentally friendly technology ${ }^{[10]}$.

\section{Characterization of TE Materials and Their Limitations}

The main disadvantage of TE is the relatively low conversion efficiency and figure-of-merit (ZT) compared to other alternative technologies ${ }^{[9]}$. The efficiency of TE materials is defined by a dimensionless parameter $Z T$ :

$Z T=\frac{S^{2} \sigma^{T}}{k}=\frac{S^{2} \sigma^{T}}{\left(k_{e l}+k_{\text {lat }}\right)}$,

where $S$ is the Seebeck coefficient, $\sigma$ is the electrical conductivity, $T$ is the absolute temperature and $k$ is the thermal conductivity, which can be divided into two parts: $k_{e l}$ and $k_{\text {lat }}$, electronic and lattice vibrations (phonons) conductivity, respectively ${ }^{[12]}$.

According to Eq. (1), to obtain a high $Z T$ value in TE materials both Seebeck coefficient and electrical conductivity must be large while thermal conductivities must be minimized. As these transport characteristics depend on material properties, some parameters have to be optimized to get optimal $Z T$ value. However, as these properties are interrelated through the physics and chemistry of the materials, it is not possible to engineer these properties separately.

Electrical conductivity of TE materials can be expressed by Eq. 2

$\sigma=n \cdot e \cdot \mu, \quad$ (2)

where $e$ is the charge of unit carrier, $n$ and $\mu$ are the concentration and mobility of charge carriers, respectively. The electrical conductivity can be significantly improved through chemical doping. Theoretical calculation showed that ideal concentration of charge carriers should be in the range of $10^{19}$ $10^{21} \mathrm{~cm}^{-3[4]}$. Simple doping of impurities can also improve the Seebeck coefficient. In semiconductors around $90 \%$ of thermal conductivity $k$ comes from $k_{\text {lat }}$. Hence, the reduction of the lattice thermal conductivity will lead to enhancement of the TE materials ${ }^{[13]}$. Glasses exhibit some of the lowest thermal conductivity compare to semiconductors. In glassy materials, thermal conductivity is viewed as a random walk of energy through a lattice rather than rapid transport via phonon, leading to the concept of a minimum thermal conductivity ${ }^{[4]}$. However, glasses have poor TE properties compared to crystalline semiconductors due to increased electron scattering and lower effective masses of the carrier. Therefore, TE effect requires a rather unusual material: a "phonon-glass electron- 
crystal" ${ }^{[14]}$, meaning the materials should have a low lattice thermal conductivity as in a glass and a high electrical conductivity as in metallic crystals.

To accelerate the pace of materials research, the Materials Genome Initiative (MGI) ${ }^{[15]}$ was launched in 2011 in USA. As illustrated in Figure 1, materials innovation infrastructure is at the core of the initiative. In line with MGI, there is a significant research into synthesizing new materials and fabricating material structures with improved TE performance, with an intention to improve the TE materials' figure-of-merit by reducing the lattice thermal conductivity [7,8]. However, in some parasitic applications, this low conversion efficiency can also be viewed as a distinct advantage ${ }^{[10]}$. Furthermore, it has been acknowledged that in situations where the supply of heat is cheap or free, as in the case of waste heat or solar energy, efficiency of the TE generating systems is not an overriding consideration ${ }^{[9]}$. Generally, development of high-efficiency TE materials for waste-heatrecovery systems is urgent and will bring vast economic and environmental benefits ${ }^{[16]}$.

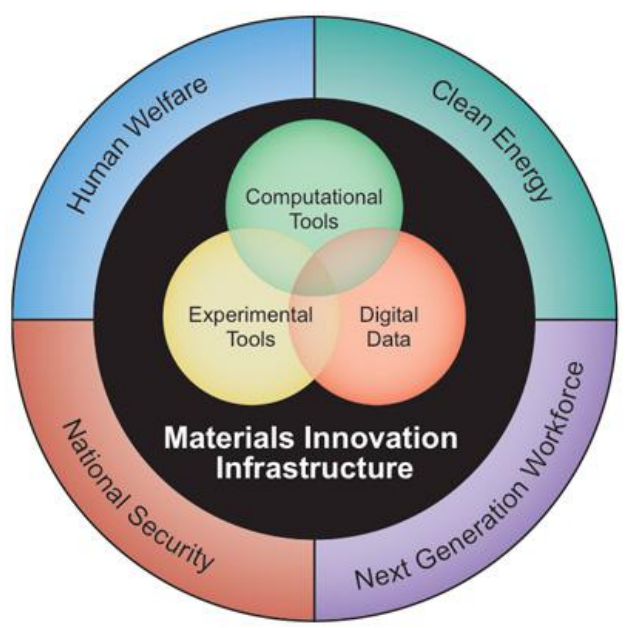

Fig.1 An Overview of the MGI ${ }^{[15]}$

\section{Advanced TE Materials}

Highly efficient TE materials that convert waste heat into electricity is a subject of increasing interest both scientifically and commercially. As shown in Figure 2, until recently, TE applications have been confined to niche applications because of this low conversion efficiency $(Z T<1)$. Since the early 1990 s, materials with $Z T>1$ have been discovered and ongoing materials research promises for the discovery of materials with even with higher $Z T$ values. Recently, new materials have been synthesized with $Z T$ being pushed to above $1.8^{[17]}$. The predicted device conversion for these materials is about $15-20 \%{ }^{[6]}$.

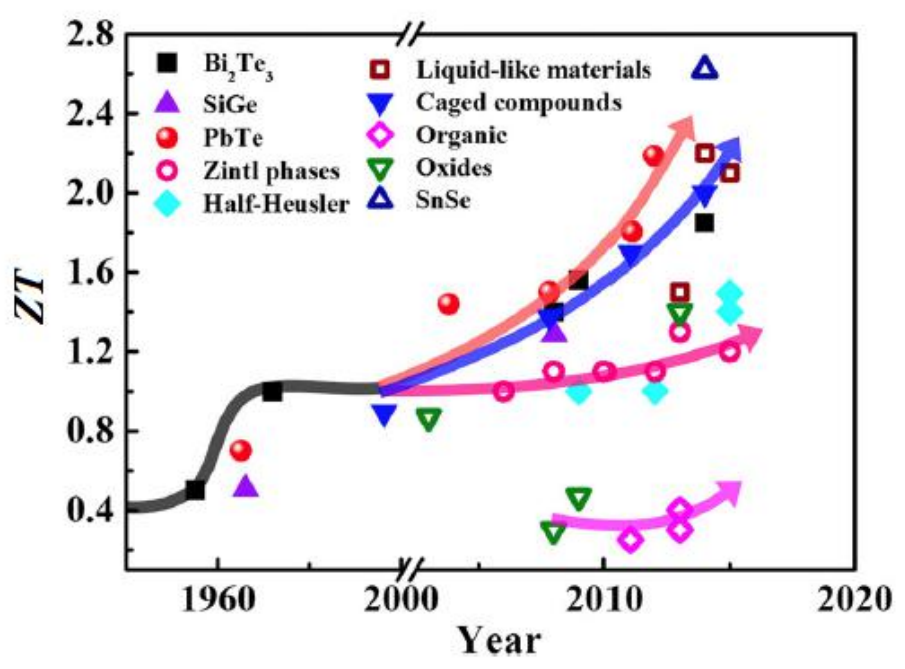

Fig.2 Timeline Underscoring the Maximum Values of $Z T$ for Typical TE Materials ${ }^{[18]}$ 
The dependence of the conversion efficiency on $Z T$ is shown in Figure 3. According to this data, $Z T$ of TE materials should be at least $\sim 3$ in order to have the device conversion efficiency, which is competitive with those of traditional power generators ${ }^{[13]}$.

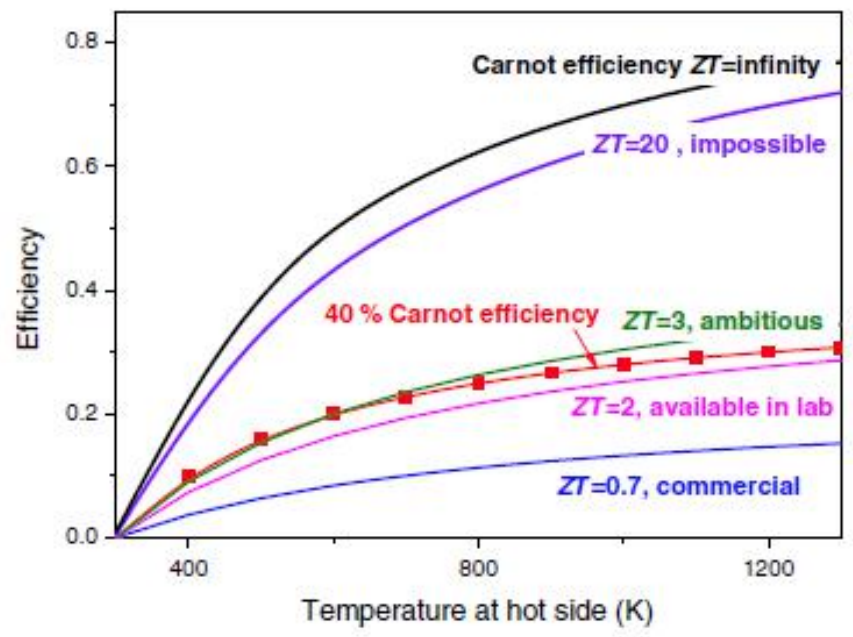

Fig.3 Conversion Efficiency of TE Materials with Changing Temperature Difference and ZT, Assuming that the Cold Side Temperature is $300 \mathrm{~K}^{[13]}$

Generally, two different approaches were applied to search for the next generation TE materials. One of these approaches is to synthesize new bulk multicomponent TE materials with complex structures and the other is to look for low dimensional TE systems ${ }^{[16,19]}$.

\subsection{Semiconductor TE Materials}

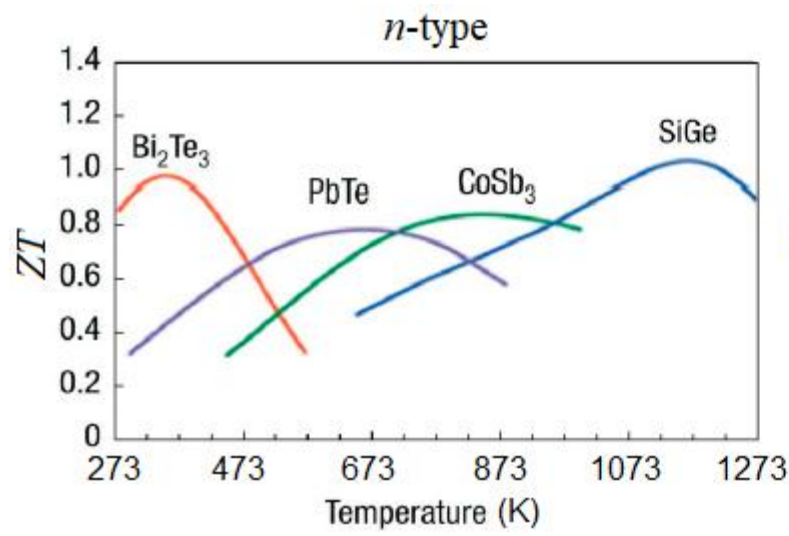

Semiconductors are one of the most used class of TE materials ${ }^{[20]}$. In the $1950 \mathrm{~s}$, the first generation of bulk TE materials, such as $\mathrm{Bi}_{2} \mathrm{Te}_{3}, \mathrm{Sb}_{2} \mathrm{Te}_{3}$, PbTe with a $Z T$ of $\sim 0.6$ at $300 \mathrm{~K}$ were developed for practical application in TE refrigerators ${ }^{[13,21]}$. Fig.4 illustrates the relative performance or figure of merits of various $n$ - and $p$-type materials over a wide range of temperatures.

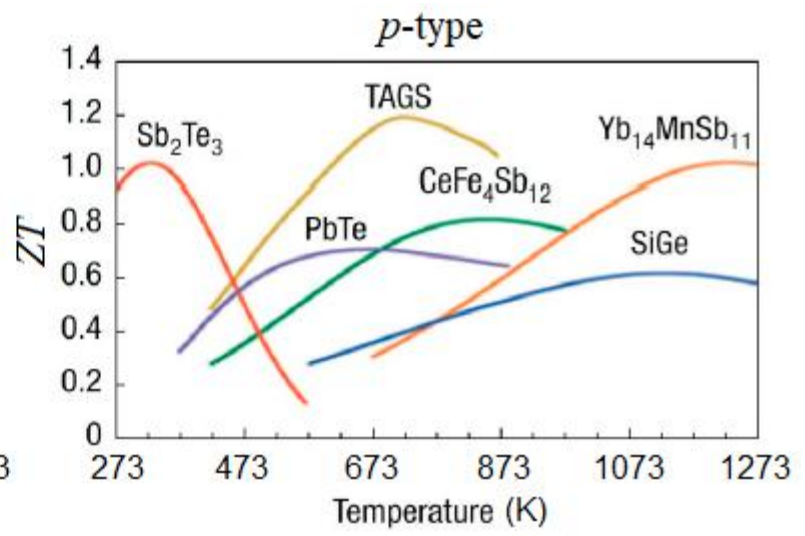

Fig.4 ZT of State-of-the-art Commercial Materials and Those Used or Being Developed by NASA for TE Power Generation: $n$-type Semiconductors (left side) and $p$-type Semiconductors (right side) ${ }^{[4]}$

The differentiation between $n$ - and $p$-type materials is important because $n$-doped compounds have a negative Seebeck coefficient whereas those $p$-doped materials have a positive one. Mixed $n$ - and $p$-type materials will lead to both charge carriers moving to the cold and canceling out the induced Seebeck voltages. Therefore, the best TE materials are semiconductors that are so heavily doped that their transport properties resemble those of metals. The maximum $Z T$ value for these materials is about 0.9 at room temperature as shown in Figure 4. Such properties allow to use $\mathrm{Bi}_{2} \mathrm{Te}_{3}$-based materials in 
small TE refrigerators ${ }^{[4,22]}$. Most TE coolers are alloys of bismuth telluride that has been suitably doped to provide individual blocks or elements having distinct $n$ - and $p$-type characters ${ }^{[23]}$. In addition to $\mathrm{Bi}_{2} \mathrm{Te}_{3}$, there are other TE materials including $\mathrm{PbTe}, \mathrm{SiGe}$, and $\mathrm{Bi}-\mathrm{Sb}$ alloys that may be used under specific conditions. In the 1960s, a slight increase of $Z T$ values were achieved by formations of solid solutions, such as $\mathrm{Bi}_{2} \mathrm{Te}_{3}-\mathrm{Sb}_{2} \mathrm{Te}_{3}, \mathrm{PbTe}-\mathrm{SnTe}$, and $\mathrm{Si}_{1-\mathrm{x}} \mathrm{Ge}_{\mathrm{x}}$. Although, point defects in the solid solutions serve to decrease the lattice thermal conductivity by increasing the scattering of heat carrying phonons, concurrent reductions in the charge carrier mobility were also observed. Therefore, the overall $Z T$ enhancement for this kind of materials is limited ${ }^{[16]}$. From the 1960 s to 1990 s, one of the best commercial material with $Z T \approx 1$ was $\left(\mathrm{Bi}_{1-\mathrm{x}} \mathrm{Sb}_{\mathrm{x}}\right)_{2}\left(\mathrm{Se}_{1-}\right.$ $\left.{ }_{\mathrm{y}} \mathrm{Te}_{\mathrm{y}}\right)_{3}{ }^{[19]}$.

Recently, many works have been conducted to apply TE thin films of $\mathrm{Bi}_{2} \mathrm{Te}_{3}, \mathrm{Sb}_{2} \mathrm{Te}_{3}$ and their solid solutions $(\mathrm{Bi}, \mathrm{Sb})_{2} \mathrm{Te}_{3}$ to microdevices, such as thermal sensors and microcoolers. $\mathrm{Bi}_{2} \mathrm{Te}_{3}$ and $\mathrm{Sb}_{2} \mathrm{Te}_{3}$ are narrow band-gap semiconductors with superior TE characters at $\sim 298 \mathrm{~K}$. Thermal sensors using the Seebeck effect have several advantages, such as high sensitivity, no offset and no need for biasing and can be utilized in various applications, including infrared sensor, microcalorimeter and RMS converter. With ever increasing power and packaging density of electronic devices, thermal management has become one of the major issues to ensure the performance and reliability of electronic devices. TE microcoolers using thin film processes can be an effective solution to thermal management of electronic devices due to their advantages, such as large cooling power density inversely proportional to the thin-film thickness, compatibility with electronic device fabrication and short response time. While various processing techniques can be used for TE thin-film fabrication, electrodeposition is attractive because it is a rapid and inexpensive process ${ }^{[23]}$.

\subsection{Superionic TE Materials}

According to Slack ${ }^{[14]}$ and Chen et al. ${ }^{[16]}$, the ideal TE materials can be described as "phonon-glass electron-crystal", which suggests that an ideal TE material should be the combination of the glass-like thermal conductivity and crystal-like electrical conductivity. This combined effect is the most achievable in bulk materials with complex crystal structures, such as superionic conductors (SIC) or solid electrolytes. Solid electrolytes are intermediate in nature between crystalline solids, which possess a regular structure with immobile ions, and liquid electrolytes, which have no regular structure with fully mobile ions. The high ionic conductivity in SIC $\left(\sim 1(\Omega \cdot \mathrm{cm})^{-1}\right.$ at elevated temperatures is usually due to lattice defects. This is in stark contrast to the normal solids, which have ionic conductivity $\sim 10^{-}$ ${ }^{8}(\Omega \cdot \mathrm{cm})^{-1}$ as a result of their substantially fixed ions. Ionic conductivity of SIC is similar to that of molten salt. At the same time, the lattice thermal conductivity (k) of SIC is extremely low. For example, in $\mathrm{Ag}_{2} \mathrm{Se}_{0.5} \mathrm{Te}_{0.5} k \approx 0.5 \mathrm{~W} / \mathrm{mK}$ at $400 \mathrm{~K}$ and in $\mathrm{Ag}_{8} \mathrm{GeTe}_{6} k \approx 0.25 \mathrm{~W} \cdot \mathrm{mK}^{-1}$ at $300 \mathrm{~K}$ with negligible contribution from electronic conductivity ${ }^{[24,25]}$. For these reasons, SIC is a good bulk material for TE applications.

Pardee and Mahan ${ }^{[26]}$ has divided SIC into three different categories. Type I SIC includes compounds such as $\mathrm{AgI}{ }^{[27]}$, for which ionic conductivity increases sharply at a phase transition temperature as shown in Figure 5. Type II includes superionic compounds such as $\mathrm{PbF}_{2}{ }^{[28]}$, which attain high levels of ionic conductivity following a gradual and continuous disordering process within the same phase. Type III superionic materials do not have a clear phase transition but achieve high ionic conductivity by increased mobility of a number of thermally activated defects. Sodium doped $\beta$-alumina variants are good examples of type III superionic materials ${ }^{[24]}$. Thermodynamic studies of SIC have shown that during the phase transitions the entropy change per atom is approximately the same as the entropy change per atom upon melting ${ }^{[30]}$. For this reason, the transition to the superionic state is called "sublattice melting". Therefore, SIC can be applied as TE materials due to the presence of pathways for ionic conductivity and their quasi-molten sublattice hinder the heat transport through transverse mode phonons ${ }^{[31]}$. The high ionic $(\sigma)$ and low thermal $(k)$ conductivities of SIC leads to a significant enhancement in $Z T$, in accordance with Eq. 1.

Superionic compounds have a big family. The crystalline silver and copper-based halide and chalcogenide compounds are a large class of SIC. As an example, Figure 6 shows the structure of hightemperature modification of $\mathrm{Ag}_{8} \mathrm{SnSe}_{6}$ compound (space group $F \overline{4} 3 \mathrm{~m}$ ) ${ }^{[32]}$. Due to the presence of excess sites for $\mathrm{Ag}^{+}$ions as well as the presence of passageways for $\mathrm{Ag}^{+}$ions, $\alpha-\mathrm{Ag}_{8} \mathrm{SnSe}_{6}$ belong to the class of superionic materials. 


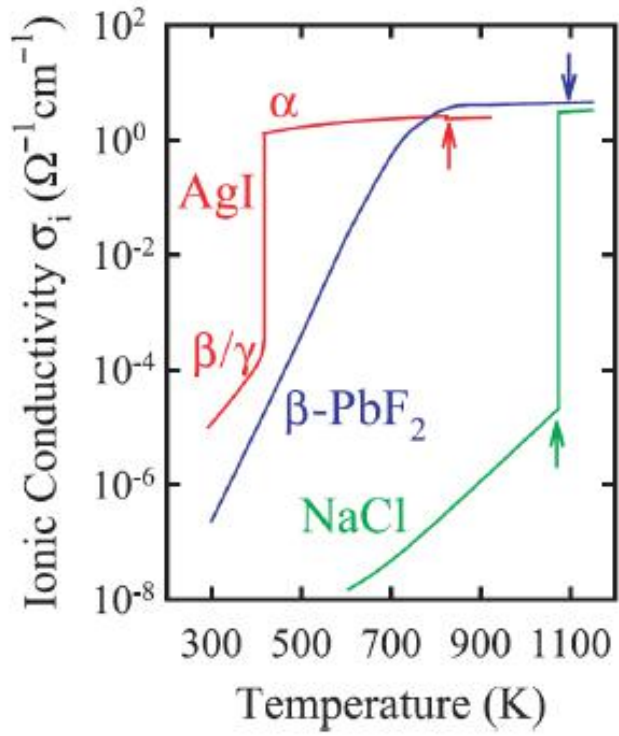

Fig.5 Ionic Conductivity of Selected Compounds ${ }^{[24,29]}$. Arrows Indicates Melting Temperatures

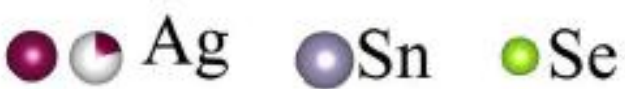

(a)

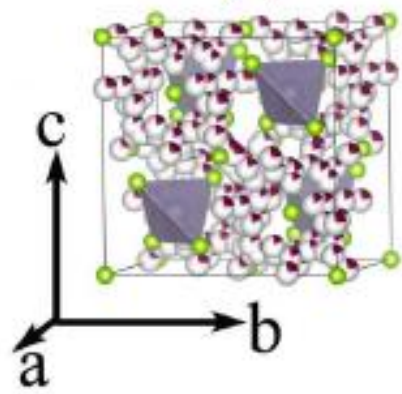

(b)

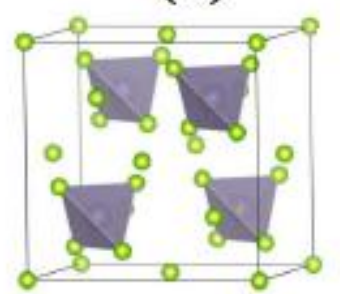

(c)

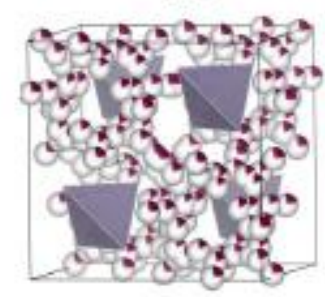

Fig.6 Cubic Structure of $\mathrm{Ag}_{8} \mathrm{SnSe}_{6}$ : (a) a unit cell, (b) anion a framework with four $\mathrm{Se}^{2-}$ and four tetrahedral $\left[\mathrm{SnSe}_{4}\right]^{4-}$ units inside the tetrahedral voids, and (c) the $\mathrm{Ag}^{+}$ions are delocalized throughout the tetrahedral voids ${ }^{[32]}$

The basic principles of effective superionic conduction were published in ${ }^{[33]}$. First of all, it must be possible for the mobile ion to move between lattice sites. Second of all, the structure must have a fully connected network of lattice sites accessible to the mobile ions with low energy barriers between them. Third of all, mobility should be enhanced through more sites for the conducting ions than there are ions to fill them.
Conductivity data for a variety of solid electrolytes are given in Figure 7 in the form of the typical Arrhenius-type diagrams. Data for concentrated $\mathrm{H}_{2} \mathrm{SO}_{4}$, a typical liquid electrolyte, are shown for comparison. The target for improved solid electrolytes is, therefore, the top right-hand corner in Figure 7, i.e. high conductivity at low temperatures. 


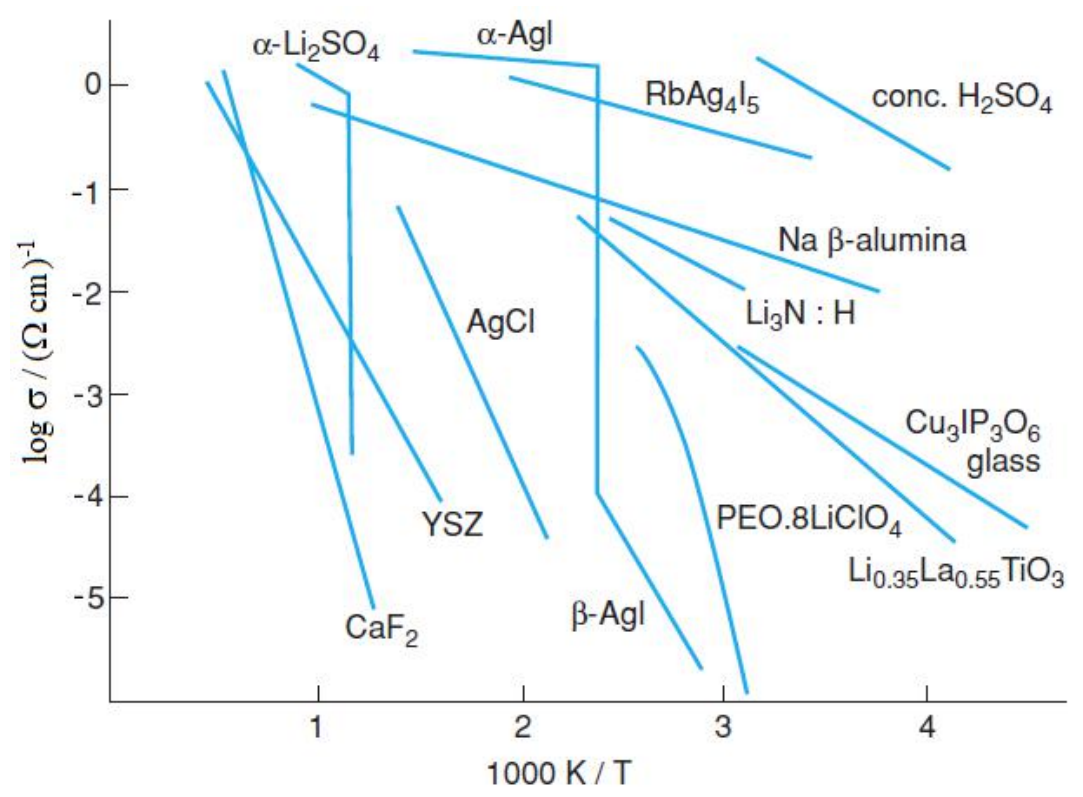

Fig.7 Ionic Conductivity of Selected Solid Electrolytes in Comparison with Concentrated $\mathrm{H}_{2} \mathrm{SO}_{4}{ }^{[23,34]}$

This problem can be solved by discovering new high ionic conductivity materials. This discovery of new compounds requires detailed investigation of phase equilibria of the corresponding system. Investigation of the phase equilibria of the multicomponent systems involves experimental difficulties. Incongruent melting of some phases, low thermal stability, kinetic hindrances to polymorphic phase transformations and change of the regions of stabilities of some phases with increasing temperature complicate the selection criteria to confirm the attainment of the equilibrium state. These experimental difficulties can partially be solved through theoretical calculations of the $T$ vs. $x$ phase diagrams using, for example, the CALPHAD methods ${ }^{[35]}$. Thermodynamic properties of some recently characterized multicomponent superionic compounds are published in ${ }^{[36-38]}$.

\subsection{Nanoscale and Nanostructured TE Materials}

Energy transport in nanostructures differs significantly from macrostructures because of classical and quantum size effects on energy carriers. Experimental results show that the thermal conductivity values of nanostructures are significantly lower than those of corresponding bulk materials. The reduction in thermal conductivity can be used to increase $Z T$ in such systems. Therefore, lowdimensional materials, such as quantum wells, superlattices, quantum wires and quantum dots offer new ways to manipulate the electron and phonon properties of a given material. In the case where quantum effects are dominant, the energy spectra of electrons and phonons can be controlled by altering the size of the structures, leading to new ways to increase $Z T$ values ${ }^{[39]}$.

The idea of selectively modifying materials properties using lower dimensional structures was introduced by Hicks and Dresselhaus ${ }^{[40]}$. They theoretically showed that by using two-, one-, and zero-dimensional structures one could obtain significant increment in electronic properties compared to the corresponding bulk materials. At the same time, nanostructures can significantly reduce thermal conductivity by enhancing the phonon scattering of materials. The theoretical calculation showed that $\mathrm{Bi}_{2} \mathrm{Te}_{3}$ with quantum well (two-dimension) or quantum wire (onedimension) structure can have the potential to reach a significantly high $Z T$ value as shown in Figure 8. It has been observed that the maximum $Z T$ increased monotonically with the decrease of characteristic length (thickness for quantum well or diameter for quantum wire) ${ }^{[41]}$. 

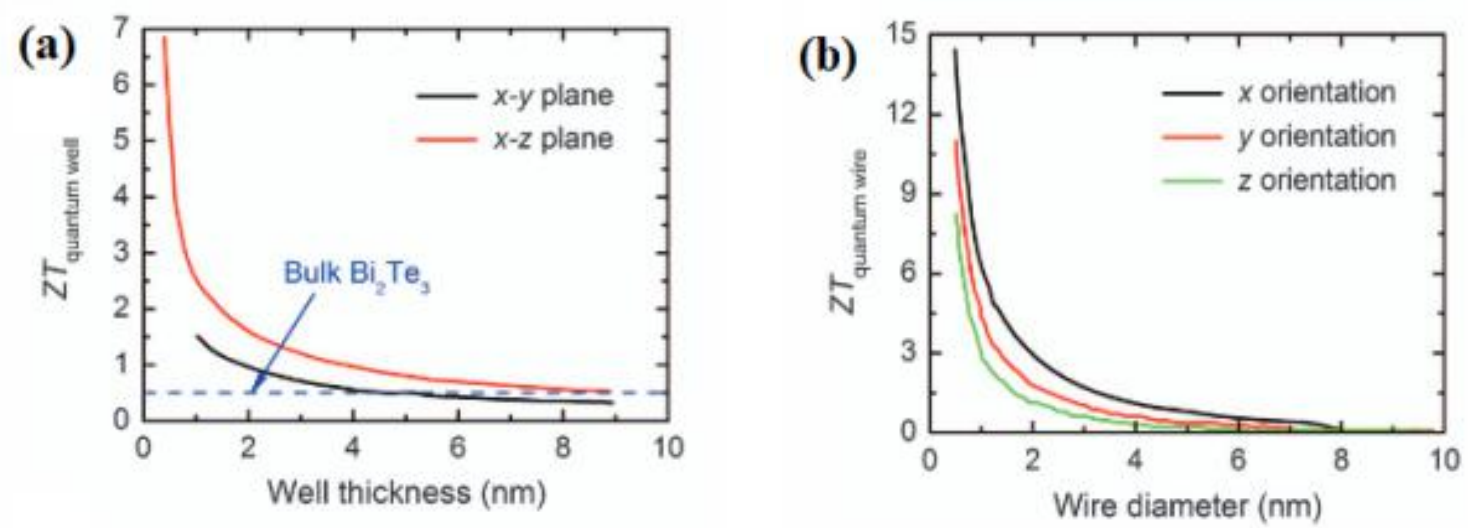

Fig.8 ZT of Quantum (a) Well as a Function of the Layer Thickness and (b) Wire as a Function of the Diameter ${ }^{[41]}$

By the investigation of $\mathrm{Bi}_{2} \mathrm{Te}_{3}-\mathrm{Sb}_{2} \mathrm{Te}_{3}$ quantum well superlattices, Venkatasubramanian et al. ${ }^{[42]}$ observed the value $Z T=2.4$ for nanostructured in contrast to bulk materials with $Z T=1.1$. Harman et al. [43] developed quantum-dot superlattices in the $\mathrm{PbTe}-$ $\mathrm{PbSeTe}$ system described as $\mathrm{PbSe}$ nanodots embedded in a $\mathrm{PbTe}$ matrix with $Z T=1.6$, which is significantly higher than their corresponding bulk material value $Z T=0.34$. Zhao et al. showed that the effective thermal conductivity of a $\mathrm{Bi}_{2} \mathrm{Te}_{3}$ nanocomposite with tubular $\mathrm{Bi}_{2} \mathrm{Te}_{3}$ nanowire inclusions leads to $25 \%$ increase in $Z T$ compared to homogenous bulk material ${ }^{[4]}$.
Further research in TE materials will focus on issues related to the reduction in thermal conductivity through the introduction of soluble second phases, mass fluctuation alloying or nanostructures such as the quantum dots ${ }^{[45]}$. The use of multiscale phonon scattering is a new strategy to minimize the thermal conductivity of TE materials. Nano-structuring has been proven to be an effective approach to enhance $Z T$ by reducing $k_{\text {lat }}$ through the placement of suitable nanoscale precipitates in the matrix. For example, Biswas et al. ${ }^{[46]}$ reduced $k_{\text {lat }}$ and increased $Z T$ value from $\sim 1.1$ to 2.2 at $915 \mathrm{~K}$ for a sample $\mathrm{PbTe}-$ $\mathrm{SrTe}(4 \mathrm{~mol} \%)$ doped with $2 \mathrm{~mol} \% \mathrm{Na}$, as illustrated in Figure 9.

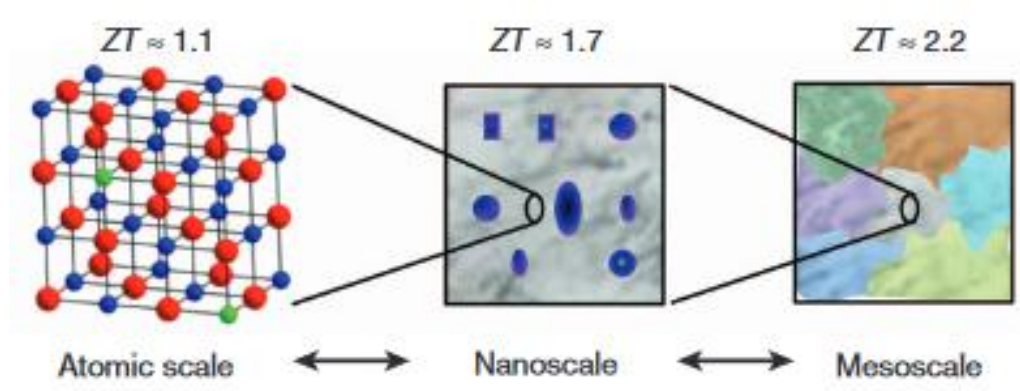

Fig.9 The Maximum ZT Values for all Length Scales of Scattering Centers (from Left to Right: the Atomic Scale, the Nanoscale, and the Mesoscale in PbTe Structure) ${ }^{[46]}$

Devices based on two-dimensional TE materials, such as thin films, quantum well, and superlattices can be used in small-scale electronic and optoelectronics applications where small heat loads or low levels of power generation are required ${ }^{[47]}$. However, fully functional practical TE coolers have not been made from these nanomaterials due to the difficulties in integrating nanoscale materials into microscale devices and packaged macroscale systems ${ }^{[16]}$.

Although, the new TE nanomaterials have high practical performance but they are encountering the following challenges [14]: (1) large-scale preparation of size, shape, and composition tunable nanomaterials; (2) poor stability of nanostructures as they can be destroyed during compression into highly dense pellets at high temperature and pressure; (3) largescale fabrication of TE devices from one dimensional nanowires and two dimensional nano-sheets is a big issue.

Current research in nanostructured composites for TE materials combines low-dimensional and bulk 
materials for TE application. According to Dresselhaus et al. [19], bulk nanostructured TE materials (BNTM) are bulk materials embedded with nanoparticles or interfaces with nanometer size. Compared to those TEs with low dimensions, bulk nanostructured TE materials can be produced in a form suitable for current TE device configuration. There are three schemes to synthesis BNTM as shown in Figure 10. One scheme is to form TE nanocomposites with single-phase nanograins, which only involves the reduction of thermal conductivity (Figure 10 (a)). The other two strategies are to form second phase nanoinclusions (Figure 10 (b) and (c)), where a large number of interfaces are formed between the TE materials and nanoinclusions ${ }^{[48]}$.
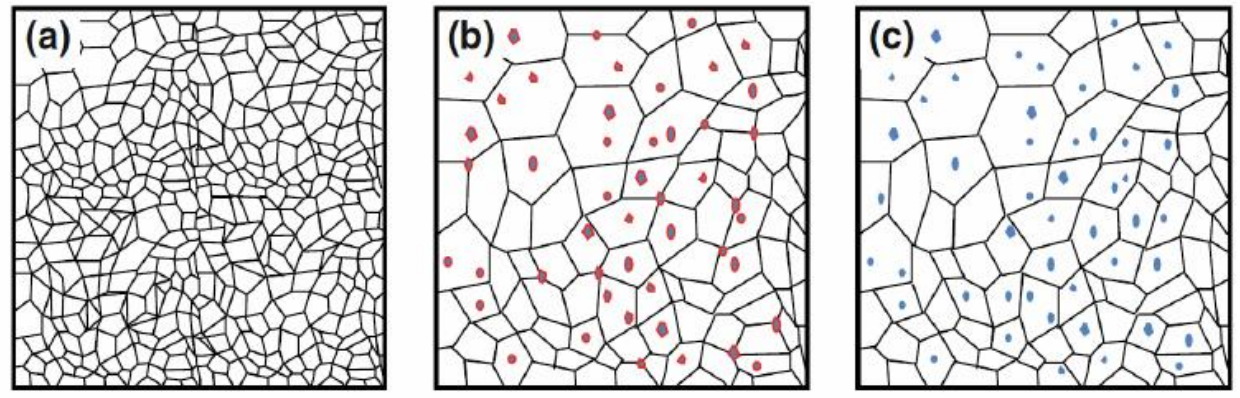

Fig.10 Approaches for Bulk TE Nanocomposites: (a) Nanograined Composite, (b) Nanoinclusion Composite with an Incoherent Interface, and (c) Nanoinclusion Composite with a Coherent Interface ${ }^{[48]}$

For example, high values of $Z T$, ranging from 1.6 at $300 \mathrm{~K}$ to 3 at $550 \mathrm{~K}$ for Bi-doped $n$-type PbSe$\mathrm{Te} / \mathrm{PbTe}$ quantum-dot super lattice samples grown by molecular beam epitaxy, are reported by Harman et al. ${ }^{[49]}$. $\mathrm{Cu}_{2} \mathrm{Se}$ nanocomposites show a very high $Z T$ of above 2.1 at $T \approx 973 \mathrm{~K}$, which is about $40 \%$ higher than its bulk counterpart ${ }^{[50]}$. Synthesis of new BNTM with higher $Z T$ values require a fundamental understanding of carrier transport in these complex materials.

\section{TE Materials and Cooling Applications in Electronic Devices}

A practical TE cooler consists of two or more elements of semiconductor materials that are connected electrically in series and thermally in parallel as shown in Figure 11. These TE elements and their electrical interconnects are typically mounted between two ceramic substrates. The substrates serve to hold the overall structure together mechanically and to insulate the individual elements electrically from one another and from external mounting surfaces. After integrating the various component parts into a module, TE modules ranging in size from approximately $2.5-50 \mathrm{~mm}^{2}$ in area and 2.5-5 $\mathrm{mm}$ in height may be constructed.

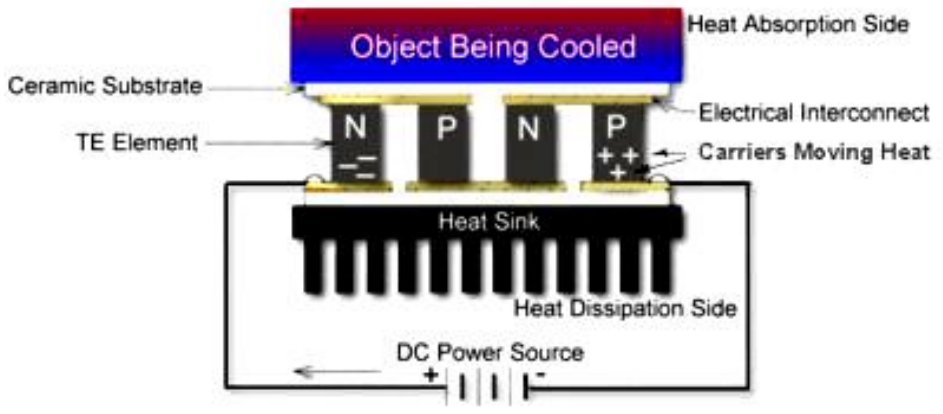

Fig.11 Schematic Diagram of a Typical TE Cooler ${ }^{[23]}$

Both $n$ - and $p$-type bismuth telluride materials are used in TE coolers. This arrangement causes heat to move through the cooler in one direction while the electrical current moves back and forth alternately between the top and bottom substrates through each $n$ - and $p$-type elements. The module illustrated in 
Figure 11 has two pairs of $n$ - and $p$-type elements and is termed as "two-couple module". $n$-type material is doped such that it will have an excess of electrons (more electrons than needed to complete a perfect molecular lattice structure) and $p$-type material is doped such that it will have a deficiency of electrons (fewer electrons that are necessary to complete a perfect lattice structure). The extra electrons in the $n$ type material and the "holes" resulting from the deficiency of electrons in the $p$-type materials are the carriers which move the heat energy through the TE material ${ }^{[8,23]}$. Figure 11 shows a typical TE cooler with heat being moved as a result of an applied electrical current $(I)$. Most of the TE cooling modules are fabricated with an equal number of $n$ - and $p$-type elements where one $n$ - and $p$-type elements pair form a TE "couple".

\section{TE Applications for Automotive Industry}

In conventional cars, about $80 \%$ of the fuel's energy is wasted as heat. TE systems could perhaps be used to generate electricity directly from this waste heat. Because the amount of fuel used for transportation is such a huge part of the global energy consumption, even a small percentage alteration could result in significant impact.

Automotive applications for TE materials are growing in importance, which are driven by the customers desire for high efficiency and for more features ${ }^{[51,52]}$. Most of TE applications can be divided into two segments. One segment is based on the Peltier effect and is focused on various applications of heating or cooling. The other segment is based on the Seebeck effect and is focused on power generation, primarily from waste heat. The heating and cooling applications are in production and further progress.

\subsection{Peltier Effect Applications}

While customers expect high efficiency from vehicles, there is a competing demand for additional features for comfort and convenience. TE materials can offer vehicle owners several unique features by providing heating and cooling for various items on the vehicle. Seats with TE heating and cooling are available today and have been in production for almost a decade for many luxury vehicles. Cup holders that keep beverages hot or cold are available on a few vehicle models. Small TE refrigerators or wine coolers are available as features for limousines and other luxury vehicles. Today, several manufacturers are producing warmer and cooler units for automotives. These units can be set to cool or heat food and drink using the 12$\mathrm{V}$ power generated from the vehicles ${ }^{[51]}$.

Many of these warmer-cooler units are portable and can be used in any location where electric power is available. All of these TE systems offer the benefit of being small, relatively lightweight, and silent in operation. These unique features make TE technology very attractive even though the efficiency is low. The most promising area for application of the Peltier effect has been discussed in numerous patents, papers and presentations but has not reached production level yet. This delay in applying for large scale commercial production has been due in part to the relatively low efficiency of TE materials (low $Z T$ and low coefficient of performance). The impact on the vehicle design has been an additional part of the delay. TE materials have the potential to revolutionize automotive heating, ventilation and air-conditioning (HVAC) systems. Improved TE materials enable the production of HVAC systems with several very desirable and unique features, such as no greenhouse gases required for refrigerant, noiseless, faster operation, especially faster delivery of warm air on cold starts, potentially lighter weight systems and increased reliability and durability due to no or few moving parts.

HVAC systems based on TE modules contribute to the acceptance of hybrid, electric or fuel cell vehicles where quiet operation without the need for mechanical input is needed. There are at least two distinctly different approaches for TE HVAC systems: direct replacement or a new distributed system ${ }^{[51]}$.

\subsection{Seebeck Effect Applications}

The global demand for oil is continuing to increase with record high prices for a barrel of oil. In most countries, the price of a liter or gallon of gasoline or diesel fuel is at record highs and increasing. There is growing concern about the effect of greenhouse gases, especially carbon dioxide emission, on the environment. All of these issues are driving the demand for obtaining more useful energy out for every gallon of fuel burned. Many new engines, transmission and vehicle technologies are being produced or are in development to make vehicle transportation more fuel efficient ${ }^{[51]}$.

All of these technologies are missing one important issue: much of the energy from a gallon of fuel will still be unusable heat in the vehicle exhaust or cooling system. TE technology presents the opportunity that can make use of this waste heat. By converting some 
of the vehicles waste heat to electric power, the electric generators mechanical load on the engine can be reduced or eliminated, which improves the vehicle fuel efficiency. An automotive TE generator that uses waste heat as its energy source consists of several components as illustrated in Figure 12.

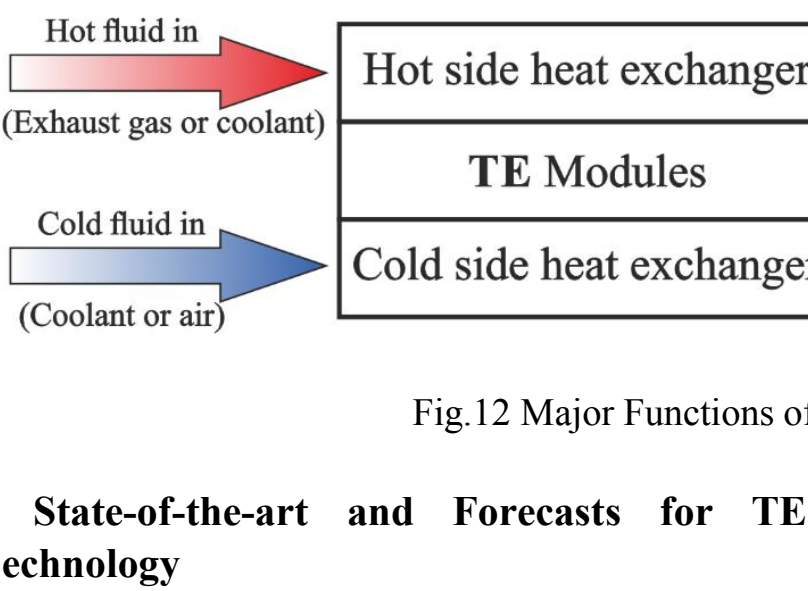

TE technology is commercially successful in cooling, refrigeration and space-craft power applications. According to Hachiuma and Fukuda [53], estimated sales of TE modules in 2006 was around \$200 million with the consumer market being the largest sector, accounting for $35 \%$ of the total market distribution followed by telecom $16 \%$ and automotive $14 \%$. Research laboratories of medical and biological markets account for $12 \%$, semiconductor processing $8 \%$, and the defense and space sector $6 \%$ of the total market [53]. Historically, the defense and space industries used to be at the center of the TE technology [53] with radioisotope TE generators having a genuinely enabling technology for deep space exploration ${ }^{[7]}$. However, this sector of the market is now flat and is not showing signs of growth. The consumer market is now the largest sector and includes applications, such as water coolers, cooler boxes and refrigerators ${ }^{[53]}$. According to Statista - the portal for statistics, the global TE modules market size in 2023 is projected to be as high as $\$ 830$ million. Today, TE coolers are widely employed in microelectronics to stabilize the temperature of laser diodes, to cool infrared detectors and charge-coupled devices [54], computer electronics and individual computer chips ${ }^{[54]}$. Recent growth can be seen in the automotive market, especially in automotive seat cooling applications ${ }^{[8,53]}$. Since 2000, TE-cooled and -heated seats were introduced into many brands of vehicles, with heated and cooled cup holders also becoming easily available ${ }^{[53]}$.

The consumer market is predicted to continue with water coolers, cooler boxes and mini-refrigerators remaining an important product applications ${ }^{[53]}$. The energy efficiency of TE refrigerators based on 38

Distributed under creative commons license 4.0
Hot fluid out

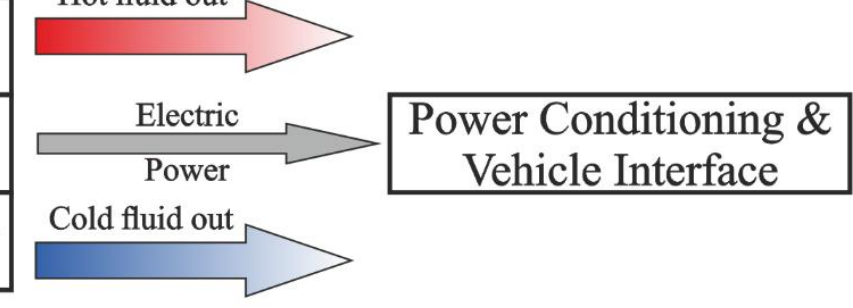

TE Generator Functions ${ }^{[51]}$

currently available materials and technologies is still lower than its counterparts. For cooling microelectronic integrated circuits, an opportunity exists to improve or replace forced-air cooling. If the computer industry is to stay on the path described by Moores Law, it is faced with the limit of forced-air cooling as microchip power and power density increases ${ }^{[55]}$. The automotive industry is seen as a potentially high volume market, and market growth exists for TE generators, specifically for waste heat recovery and the replacement of batteries in small remote power sources ${ }^{[53]}$. The possibility of using TE technology to convert waste heat into electrical power has been considered for some time. TE solutions for self-powered space and water heating have shown that the electricity generated by a TE power unit is sufficient to power all the electrical components for a residential central heating system ${ }^{[56]}$. In some areas of the developing world, the electric power supply is unreliable and intermittent, and it is common to find wood or diesel fired stoves. Studies have demonstrated that acceptable economic performance for stove-top TE generators, and a domestic woodstove and TE generator system can be achieved at a low cost with minimal complexity. Opportunities exist to implement the technology in energy harvesting or scavenging systems, for example, commercial autonomous sensors are mainly powered by primary batteries that need to be replaced, hence, it can become the largest and most expensive part of the system. Exploiting temperature differences available in nature and in/on artificial objects can be used to power autonomous devices, and TE power generators that use body heat as an energy source are appropriate for powering low-energy consumers ${ }^{[57]}$. The Seiko ThermicTM wristwatch is a good example. The need for lightning the battery burden in present and future military systems is a focus for implementing TE 
technology within the US Army, and a market exists for TE air-conditioners in submarines [58]. Furthermore, unmanned systems are being developed for reconnaissance and surveillance activities, with energy scavenged in-the-field extending the endurance and range of electric-powered unmanned systems ${ }^{[59]}$. An interesting problem potentially facing the TE industry is the availability of raw materials. There are four main raw materials used in $\mathrm{TE}$ elements, including the scarce elements $\mathrm{Te}, \mathrm{Bi}, \mathrm{Sb}$ and Se. According to Hachiuma and Fukuda ${ }^{[53]}$, these are regarded as rare materials and their supply may be limited in the future, highlighting the need to develop other types of TE materials. Contemporary problems surrounding climate change will continue to act as a stimulus for the further development of TE technology ${ }^{[60]}$.

\section{Conclusions}

TE technology can be used in power generation, heating and cooling applications. They potentially offer significant energy saving through waste heat recovery and augmented cooling. Commercial success has been achieved in cooling, refrigeration and space-craft power, with the consumer market currently the largest sector. The need for thermal management, performance and reliability for the everincreasing power and packaging density of electronic devices will increase the demand for TE technologies. However, improving the efficiency of the TE materials (for example: high temperature stability, higher Seebeck coefficients, etc.) and to secure the availability of raw materials that form TE materials need to be addressed.

In the near future, the potentially large-scale application is predicted to be in the automotive industry. Recent advances in TE materials and manufacturing techniques will provide new opportunities for TE power generation. Contemporary problems surrounding climate change will also act as a stimulus for the development of TE technologies and eventually new applications may emerge. Superionic TE materials are good candidates for the next generation of TE technology applications.

Different TE materials were synthesized by several researchers with high $Z T$ values. However, most of these materials were not commercialized due to the difficulties in preparation as well as high fabrication cost and low thermal stabilities. To improve the overall energy conversion efficiency, TE devices can be combined with other green energy generating devices such as solar cells. The development of hybrid devices involving TE devices and solar cells could be the key to solving problems related to large scale commercialization.

Further studies are required to establish a correlation between a structure, conductivity and thermal properties of TE materials to ultimately optimize their characters. Discoveries of new TE materials of high efficiency and cost effective require deeper understanding of the relationship between atoms, chemical bonds and crystalline structures as well as the electron-phonon transport phenomena in solids.

\section{Acknowledgments}

The authors are grateful to the Academy of Finland for financial support. This work was made under the project "Thermodynamic investigation of complex inorganic material systems for improved renewable energy and metals production processes" as part of the activities of the Johan Gadolin Process Chemistry Centre at Åbo Akademi University.

\section{References}

[1] Chu S, Majumdar A. Opportunities and challenges for a sustainable energy future [J]. Nature, 2012, 488: 294-303.

[2] Minnich AJ, Dresselhaus MS, Ren ZF, et al. Bulk nanostructured thermoelectric materials: current research and future prospects [J]. Energy \& Environmental Science, 2009, 2: 466-479.

[3] He W, Zhang G, Zhang X, et al. Recent development and application of thermoelectric generator and cooler [J]. Applied Energy, 2015, 143: 1-25.

[4] Snyder GJ, Toberer ES. Complex thermoelectric materials [J]. Nature Materials, 2008, 7: 105-114.

[5] Radioisotope Power Systems, viewed February 2018, https://rps.nasa.gov/

[6] Zhang X, Zhao L-D. Thermoelectric materials: Energy conversion between heat and electricity [J]. Journal of Materiomics, 2015, 1: 92-105.

[7] Gould CA, Shammas NYA, Grainger S, et al. A comprehensive review of thermoelectric technology, micro-electrical and power generation properties. In Microelectronics. 26th International Conference IEEE, 2008: 329-332.

[8] Zhang PX, Zhang GY, Lin CT, et al. New thermoelectric materials and new applications [J]. Egyptian Journal of Solids, 2004, 27: 1-7. 
[9] Riffat S, Ma X. Thermoelectrics: a review of present and potential applications [J]. Applied Thermal Engineering, 2003, 23: 913-935.

[10] Alaoui, C, Salameh, ZM. Solid state heater cooler: design and evaluation. In Power Engineering, Large Engineering Systems Conference on IEEE, 2001: 139-145.

[11] Lim S-K, Kim M-Y, Oh T-S. Thermoelectric properties of the bismuth-antimony-telluride and the antimony-telluride films processed by electrodeposition for microdevice applications [J]. Thin Solid Films, 2009, 517: 4199-4203.

[12] Goldsmid HJ. Applications of Thermoelectricity. 1960, Methuen, London.

[13] Han C, Li Z, Dou S. Recent progress in thermoelectric materials $[\mathrm{J}]$. Chinese Science Bulletin, 2014, 59: 2073-2091.

[14] Slack GA. New materials and performance limits for thermoelectric cooling. In Handbook of Thermoelectrics. 1995, Rowe DM, CRC Press, 407-440.

[15] Materials Genome Initiative for Global Competitiveness, Executive office of the president, National Science and Technology Council, Washington, USA, 2011: 1-18, viewed February 2018, https://obamawhitehouse.archives.gov/mgi

[16] Chen Z-G, Han G, Yang L, et al. Nanostructured thermoelectric materials: current research and future challenge [J]. Progress in Natural Science: Materials International, 2012, 22: 535-549.

[17] Hsu KF. Cubic AgPbmSbTe $2+m$ : bulk thermoelectric materials with high figure of merit $[\mathrm{J}]$. Science, 2004, 303: 818-821.

[18] Shi X, Chen L, Uher C. Recent advances in high-performance bulk thermoelectric materials [J]. International Materials Reviews, 2016, 61: 379-415.

[19] Dresselhaus MS, Chen G, Tang MY, et al. New directions for low-dimensional thermoelectric materials [J]. Advanced Materials, 2007, 19: 1043-1053.

[20] Lukowicz MV, Abbe E, Schmiel T, et al. Thermoelectric generators on satellites - an approach for waste heat recovery in space $[\mathrm{J}]$. Energies, 2016, 9: 541(1-14).

[21] Rosi FD. Thermoelectricity and thermoelectric power generation $[\mathrm{J}]$. Solid-State Electronics, 1968, 11: 833-868.

[22] Mi W, Qiu P, Zhang T, et al. Thermoelectric transport of Se-rich $\mathrm{Ag}_{2} \mathrm{Se}$ in normal phases and phase transitions [J]. Applied Physics Letters, 2014, 104: 133903.

[23] Ferrotec. Pure precision, Thermoelectric Technical Reference - Basic Principles of

Thermo-electric Materials, viewed February 2018, https://thermal.ferrotec.com

[24] Brown DR. Enhanced thermoelectric performance at the superionic phase transitions of mixed ion-electron conducting materials [D]. California Institute of Technology.

[25] Drymiotis F, Day TW, Brown DR, et al. Enhanced thermoelectric performance in the very low thermal conductivity $\mathrm{Ag}_{2} \mathrm{Se}_{0.5} \mathrm{Te}_{0.5} \quad[\mathrm{~J}]$. Applied Physics Letters, 2013, 103: 143906.

[26] Pardee WJ, Mahan GD. Disorder and ionic polarons in solid electrolytes [J]. Journal of Solid State Chemistry, 1975, 15: 310-324.

[27] Burley G. Structure of hexagonal silver iodide [J]. The Journal of Chemical Physics, 1963, 38: 2807-2812.

[28] Hull S, Berastegui P, Eriksson SG, et al. Crystal structure and superionic conductivity of doped with KF [J]. Journal of Physics: Condensed Matter, 1998, 10: 8429-8446.

[29] Hull S. Superionics: crystal structures and conduction processes [J]. Reports on Progress in Physics, 2004, 67: 1233-1314.

[30] Boyce JB, Huberman BA. Superionic conductors: transitions, structures, dynamics [J]. Physics Reports, 1979, 51: 189-265.

[31] Bailey TP, Uher C. Potential for superionic conductors in thermoelectric applications [J]. Current Opinion in Green and Sustainable Chemistry, 2017, 4: 58-63.

[32] Li L, Liu Y, Dai J, et al. High thermoelectric performance of superionic argyrodite compound $\mathrm{Ag}_{8} \mathrm{SnSe}_{6}[\mathrm{~J}]$. Journal of Materials Chemistry C, 2016, 4: 5806-5813.

[33] Keen DA. Disordering phenomena in superionic conductors [J]. Journal of Physics: Condensed Matter, 2002, 14: R819-R857.

[34] West AR Solid State Chemistry and its Applications. 2014, John Wiley \& Sons.

[35] Ipser H, Mikula A, Katayama I. Overview: the emf method as a source of experimental thermodynamic data [J]. Calphad, 2010, 34: 271-278.

[36] Tesfaye F, Lindberg D. Thermochemical properties of selected ternary phases in the Ag-Bi-S system [J]. Journal of Material Science, 2016, 51: 5750-5759.

[37] Moroz M, Tesfaye F, Demchenko P, et al. Determination of the thermodynamic properties 
of the $\mathrm{Ag}_{2} \mathrm{CdSn}_{3} \mathrm{~S}_{8}$ and $\mathrm{Ag}_{2} \mathrm{CdSnS}_{4}$ phases in the $\mathrm{Ag}-\mathrm{Cd}-\mathrm{Sn}-\mathrm{S}$ system by the solid-state electrochemical cell method $[\mathrm{J}]$. The Journal of Chemical Thermodynamics, 2018, 118: 255-262.

[38] Moroz M, Tesfaye F, Prokhorenko M, et al. Thermodynamic properties of superionic phase $\mathrm{Ag}_{4} \mathrm{HgSe}_{2} \mathrm{I}_{2}$ determined by the EMF method [J]. Journal of Phase Equilibria and Diffusion, 2018, 39: 11-16.

[39] Yang RG, Gang C. Nanostructured thermoel-ectric materials: from superlattices to nanocomposites [J]. Materials Integration, 2005, 18: 3136.

[40] Hicks LD, Dresselhaus MS. Thermoelectric figure of merit of a one-dimensional conductor [J]. Physical Review B, 1993, 47: 16631-16634.

[41] Mao J, Liu Z, Ren Z. Size effect in thermoelectric materials [J]. Quantum Materials, 2016, 1: 16028-16030.

[42] Venkatasubramanian R, Siivola E, Colpitts T, et al. Thin-film thermoelectric devices with high room-temperature figures of merit [J]. Nature, 2001, 413: 597-602.

[43] Harman TC. Quantum dot superlattice thermoelectric materials and devices [J]. Science, 2002, 297: 2229-2232.

[44] Zhao XB, Ji XH, Zhang YH, et al. Bismuth telluride nanotubes and the effects on the thermoelectric properties of nanotube-containing nanocomposites [J]. Applied Physics Letters, 2005, 86: 062111.

[45] Xiao C, Xu J, Li K, et al. Superionic phase transition in silver chalcogenide nanocrystals realizing optimized thermoelectric performance [J]. Journal of the American Chemical Society, 2012, 134: 4287-4293.

[46] Biswas K, He J, Blum ID, et al. High-performance bulk thermoelectrics with all-scale hierarchical architectures [J]. Nature, 2012, 489: 414418.

[47] Chowdhury I, Prasher R, Lofgreen K, et al. Onchip cooling by superlattice-based thin-film thermoelectrics [J]. Nature Nanotechnology, 2009, 4: $235-238$.

[48] Koumoto K, Mori T Thermoelectric Nanomaterials. 2013, Springer Berlin Heidelberg, Berlin, Heidelberg.
[49] Harman TC, Walsh MP, laforge BE, et al. Nanostructured thermoelectric materials [J]. Journal of Electronic Materials, 2005, 34: L19-L22.

[50] Gahtori B, Bathula S, Tyagi K, et al. Giant enhancement in thermoelectric performance of copper selenide by incorporation of different nanoscale dimensional defect features [J]. Nano Energy, 2015, 13: 36-46.

[51] Yang J, Stabler FR. Automotive applications of thermoelectric materials [J]. Journal of Electronic Materials, 2009, 38: 1245-1251.

[52] Nandhakumar I, White NM, Beeby S. (eds). Thermoelectric Materials and Devices. 2016, Royal Society of Chemistry.

[53] Hachiuma H. and Fukuda K. Activities and Future Vision of Komatsu Thermo modules, 2007, viewed February 2018, http://ect2007.its.org/ect2007.its.org/system/files lu1/pdf/01.pdf

[54] Mikalauskis GS. Selecting a thermoelectric cooler [J]. Electronic Products, 2004, 31-35.

[55] Sharp J, Bierschenk J, Lyon HB. Overview of solid-state thermoelectric refrigerators and possible applications to on-chip thermal management [J]. Proceedings of the IEEE, 2006, 94: 16021612.

[56] Qiu K, Hayden ACS. Integrated thermoelectric generator and application to self-powered heating systems. In Thermoelectrics, ICT'06. 25th International Conference, 2006, 198-203.

[57] Weber J, Potje-Kamloth K, Haase F, et al. Coinsize coiled-up polymer foil thermoelectric power generator for wearable electronics [J]. Sensors and Actuators A: Physical, 2006, 132: 325-330.

[58] Riffat S, Qiu G. Comparative investigation of thermoelectric air-conditioners versus vapour com-pression and absorption air-conditioners [J]. Applied Thermal Engineering, 2004, 24: 19791993.

[59] Thomas JP, Qidwai MA, Kellogg JC. Energy scavenging for small-scale unmanned systems [J]. Journal of Power Sources, 2006, 159: 1494-1509.

[60] Vining CB. ZT 3.5: Fifteen Years of Progress and Things to Come. Proceedings of the 5th European Conference on Thermoelectrics, ECT2007, 2007, viewed February 2018, http://ect2007.thermion-company.com ect 2007.its.org/index.html 